\title{
Malignant Mönckeberg medial calcific sclerosis
}

\author{
Isabella Pisani ${ }^{1} \cdot$ Alessandro De Troia $^{2} \cdot$ Landino Allegri $^{1} \cdot$ Domenico Corradi $^{1} \cdot$ Augusto Vaglio $^{3}$
}

Received: 4 January 2018 / Accepted: 17 January 2018 / Published online: 23 January 2018

(c) SIMI 2018

Mönckeberg medial calcific sclerosis (MCS) refers to a calcification of the medial arterial layer that is most found in the muscular arteries of the extremities and occasionally in visceral arteries [1].

We describe the case of a 50-year-old woman hospitalized because of acute ischemic lesions, involving her left lower limb, that developed over a few days. She had no conventional risk factors for atherosclerosis, and laboratory tests revealed severe renal insufficiency (serum creatinine $12.8 \mathrm{mg} / \mathrm{dl}$ ), hyperparathyroidism (serum parathyroid hormone, PTH, $1559 \mathrm{ng} / \mathrm{l}$, normal 10-60), and an elevated calcium-phosphate product (Ca $9.2 \mathrm{mg} / \mathrm{dl}, \mathrm{P} 12.4 \mathrm{mg} / \mathrm{dl}$ ). All common causes of renal failure were ruled out as well as paraneoplastic syndromes and autoimmune diseases. $\mathrm{Ca}-\mathrm{P}$ metabolism was normalized using calcimimetics and calcium-free phosphate binders. Imaging studies, with $\mathrm{X}$-ray, computed tomography (CT) and angiography, showed widespread vascular calcifications involving vertebral, renal, ovarian, hepatic, splenic, visceral, and peripheral arteries of the upper and lower limbs without overt organ or soft tissue calcifications (Fig. 1a-c). Because of the rapid progression of ischemic lesions, multiple amputations involving both lower limbs and hands became necessary. The histological examination of surgical specimens showed smallto-medium-sized muscular arteries displaying dystrophic medial calcifications that were focal or circumferential in the affected tunica media, causing luminal narrowing or sub-occlusion (Fig. 1d-f). There were no signs of atherosclerosis, and a diagnosis of MCS was made. Parathyroid

Domenico Corradi and Augusto Vaglio share senior authorship.

Augusto Vaglio

augusto.vaglio@virgilio.it

1 Department of Medicine and Surgery, University of Parma, Parma, Italy

2 Department of Medicine and Surgery, Unit of Vascular Surgery, University of Parma, Parma, Italy

3 Unit of Nephrology, University Hospital of Parma, Via Gramsci 14, 43216 Parma, Italy ultrasound and scintigraphy revealed diffuse enlargement and hypermetabolism of the four glands. Total parathyroidectomy was performed, and histological examination of the excised glands showed diffuse hyperplasia. After parathyroidectomy, no new lesions appeared. There was a progressive healing at the sites of amputation.

Chronic kidney disease (CKD) is known to be associated with bone and mineral disorders that present with laboratory alterations in $\mathrm{Ca}-\mathrm{P}$ metabolism and with vascular calcifications. Vascular calcifications are not simply due to $\mathrm{Ca}-\mathrm{P}$ precipitation, but result from an active, highly regulated process in which vascular smooth muscle cells (VSMCs) undergo apoptosis and are transformed into osteoblastlike cells involved in the mineralization process $[1,2]$. The prevalence of vascular calcification of the media ( $\mathrm{VCm}$ ) of MCS type is estimated to be $\sim 0.5 \%$ in adults, with the highest prevalence in type 2 diabetes patients. ESRD (end-stage renal disease) patients with $\mathrm{VCm}$ have less conventional risk factors for atherosclerosis, greater prevalence of $\mathrm{Ca}-\mathrm{P}$ disorders, and a longer time on hemodialysis [3]. MCS is commonly observed in muscle-type conduit arteries, and is usually revealed in the arteries of the extremities, whereas a systemic distribution with involvement of visceral arteries appears uncommon [1].

Four stages of lesion progression are recognized in MCS. In stage 1, calcifications appear as irregular deposits within the media, they are both intracellular and extracellular: the first located in VSMCs, the second associated with damaged and fractured elastic fibers in the extracellular matrix. Then, calcifications may progress to involve up to three quadrants of the cross section (stage 2) or the entire vascular circumference (stage 3), distorting the architecture of the media. In stage 4 , foci of bone formation may be found within the arterial media until they undergo osseous metaplasia giving rise to true bony trabeculae [3]. In our patient, MCS had a unique course because of the widespread distribution of vascular calcifications and the absence of classical risk factors for this complication, except for ESRD.

The literature reports usually describe the involvement of arteries of the limbs; when other kinds of arteries are 
Fig. 1 a Calcifications of splenic artery and its branches (arrowheads) assessed by abdominal CT scans without contrast medium; b calcifications of uterine arteries assessed by abdominal CT scans without contrast medium; c X-ray of hands and forearms with calcification of radial and interdigital arteries (arrowheads); amputations of the third and the fourth finger of the right hand; d Histopathological detail of an artery showing the dystrophic calcification (arrow) which involves almost the entire arterial wall profile. The arrowhead indicates the narrowed new lumen (original magnification $\times 20$, scale bar is $150 \mu \mathrm{m}$ ); e vascular wall of some deep arteries had undergone diffuse calcification and subsequent osseous metaplasia (arrow) with either complete obstruction or a virtual lumen (arrowhead) (original magnification $\times 4$, scale bar is $1 \mathrm{~mm})$; $\mathbf{f}$ mediumpower view of an artery with mild-to-moderate changes displaying wall calcification (arrow) which originates from the region of the internal elastic lamina (arrowhead) (original magnification $\times 10$, scale bar is $300 \mu \mathrm{m})$
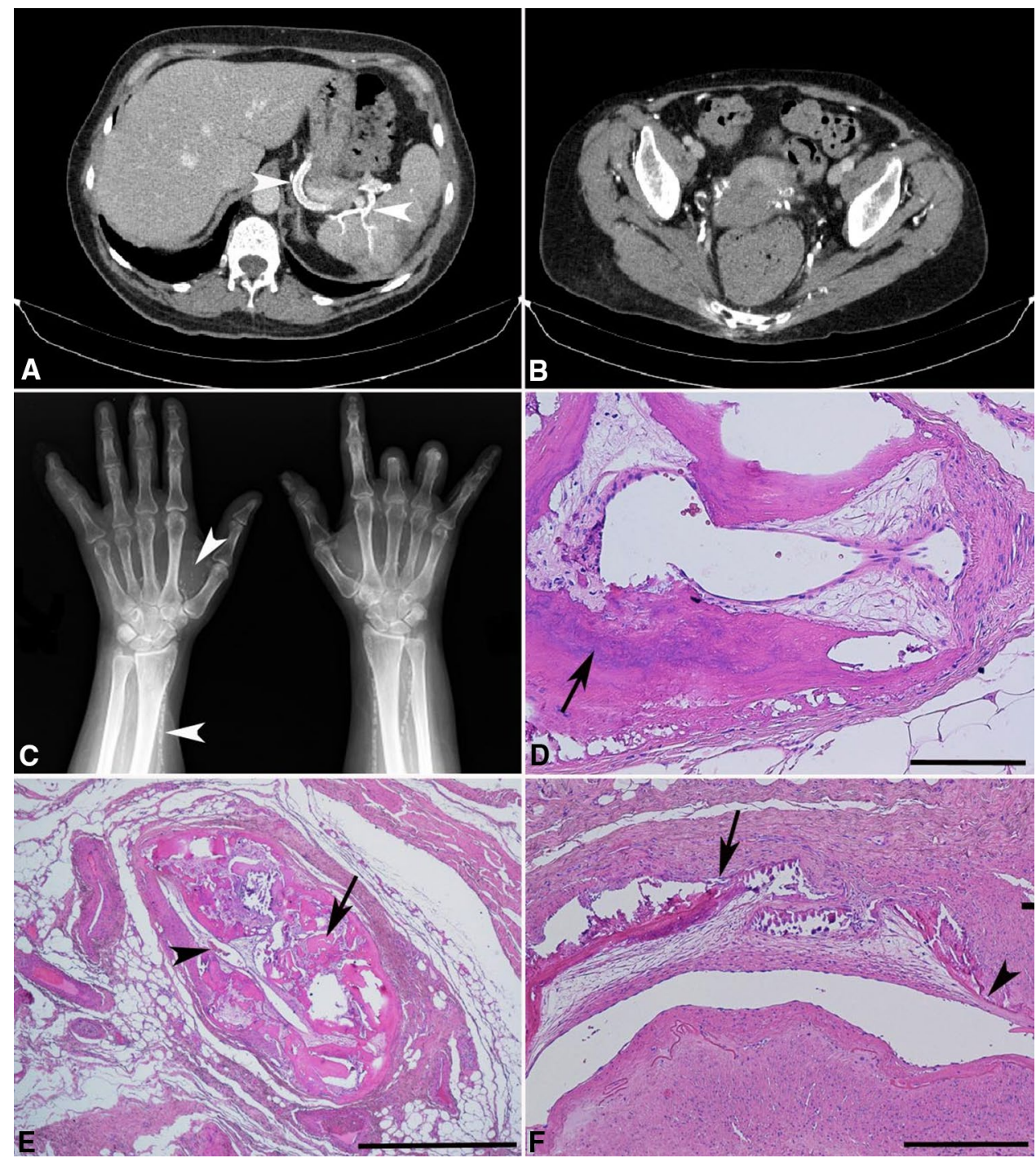

affected, other underlying disorders can be recognized (e.g., atherosclerosis, vasculitis) [3]. Our patient was only 50 years old and had no diabetes or atherosclerotic risk factors. All potential conditions predisposing to vascular calcifications (vitamin-K or coagulation disorders, osteoporosis, rheumatoid arthritis, and other autoimmune diseases) were excluded.

The only risk factors were ESRD and secondary hyperparathyroidism. VCm is very common in CKD, and its incidence increases with renal function worsening, CKD duration, and especially time on hemodialysis [1,2]. Our patient was not yet on dialysis when ischemic lesions appeared; although we cannot date the onset of her kidney disease, such a widespread vascular calcification in the literature is usually associated with years of hemodialysis dependence [3]. A number of non-traditional risk factors are associated with vascular calcifications in CKD, in particular $\mathrm{Ca}-\mathrm{P}$ metabolism abnormalities and high PTH levels. Although in vitro studies demonstrate that high phosphate levels induce mineralization in VSMCs, it is also reported that serum from uremic patients induces calcification regardless of phosphate content. Moreover, although PTH may function as a mediator of pathological calcification, it seems to prevent calcifications in VSMCs. Therefore, neither phosphate levels alone nor PTH explain the increased incidence of vascular calcifications in CKD, and the factors promoting this disease in the "uremic serum" remain under investigation $[1,2,4]$. The control of our patient's $\mathrm{Ca}-\mathrm{P}$ balance and hyperparathyroidism was achieved using the recommended drugs, but this did not stop the progression of ischemic lesions; only parathyroidectomy was successful, but in the published literature, this surgical approach does not always provide sustained benefit. Beyond the extension of MCS, another unusual characteristic of our patient's disease was its dramatic and quick course that evolved within a few days from the presence of ischemic pain to the development of 
lesions, and in about 6 months, from circumscribed lesions to multiple amputations of limbs.

It has been suggested that two types of MCS may exist, with clinical and histological differences: the first is a benign, slowly progressive, and essentially asymptomatic form with thin medial calcifications and little or no narrowing of the arterial lumen; the second is a malignant, rapidly progressive form in which massive and extensive medial calcifications may displace the internal elastic lamina toward the lumen causing luminal narrowing [5].

In summary, MCS can be a widespread and malignant disease affecting peripheral and visceral arteries, and may develop even in the absence of typical risk factors such as diabetes and a long history of hemodialysis.

Funding There was no specific funding for this article

\section{Compliance with ethical standards}

Conflict of interest The authors declare that they have no conflict of interest.

Statement of human and animal rights This article does not contain any experimental studies with human participants or animals per- formed by any of the authors. All procedures performed in this case study were in accordance with the ethical standards of our institutional research committee and with the 1964 Helsinki Declaration and its later amendments or comparable ethical standards.

Informed consent Informed consent was obtained from the patient whose clinical picture was reported in the study.

\section{References}

1. Covic A, Kanbay M, Voroneanu L et al (2010) Vascular calcification in chronic kidney disease. Clin Sci (Lond) 119(3):111-121

2. Byon $\mathrm{CH}$, Chen Y (2015) Molecular mechanisms of vascular calcification in chronic kidney disease: the link between bone and the vasculature. Curr Osteoporos Rep 13(4):206-215

3. Lanzer P, Boehm M, Sorribas V et al (2014) Medial vascular calcification revisited: review and perspectives. Eur Heart J 35(23):1515-1525

4. Johnson RC, Leopold JA, Loscalzo J (2006) Vascular calcification: pathobiological mechanisms and clinical implications. Circ Res 99(10):1044-1059

5. Meema HE, Oreopoulos DG, Rapoport A (1987) Serum magnesium level and arterial calcification in end-stage renal disease. Kidney Int 32(3):388-394 